\title{
The Electric Screening Mass in Scalar Electrodynamics at High Temperature
}

\author{
Jens O. Andersen \\ Institute of Physics \\ University of Oslo \\ P.O. BOX 1048, Blindern \\ N-0316 Oslo, Norway
}

\begin{abstract}
We study the screening of static electric fields in massless scalar electrodynamics at high temperature and zero chemical potential. Effective field theory methods are used to separate the contributions from the momentum scales $T$ and $e T$ to the electric screening mass. The effects of the distance scale $1 / T$ are encoded in the parameters of an effective three-dimensional field theory. The parameters of the effective Lagrangian can be written as a power series in $e^{2}$. The contribution to physical quantities from the scale $1 / e T$ can be calculated from perturbation theory in the effective theory and is an expansion in $e$ starting at $e^{3}$. The electric screening mass squared is computed to order $e^{4}$.
\end{abstract}

PACS number(s): 11.10.Wx, 12.20.DS

\section{Introduction}

One interesting phenomenon in hot plasmas is the screening of static electric fields at long distances. The potential between two static charges in the plasma is normally derived in linear response theory [1], and reads

$$
V(R)=Q_{1} Q_{2} \int \frac{d^{3} k}{(2 \pi)^{3}} e^{i \mathbf{k R}} \frac{1}{k^{2}+\Pi_{00}(0, \mathbf{k})} .
$$

Here, $\Pi_{\mu \nu}\left(k_{0}, \mathbf{k}\right)$ is the photon polarization tensor. In the limit $R \rightarrow \infty$, the potential is dominated by the pole in photon propagator. At leading order this pole is given by the infrared limit of $\Pi_{00}(0, \mathbf{k})$, and the potential is thus a modified Coulomb potential with an inverse screening length or electric screening mass $\Pi_{00}(0, \mathbf{k} \rightarrow 0)$. This has lead one to define the electric screening mass as the infrared limit of the polarization tensor $[\mathbb{1}$ :

$$
m_{s}^{2}=\Pi_{00}(0, \mathbf{k} \rightarrow 0)
$$


This definition cannot be the correct one, since, beyond leading order in the coupling, it is gauge-fixing dependent in non-Abelian theories $[2,3]$. Although $\Pi_{\mu \nu}\left(k_{0}, \mathbf{k}\right)$ is a manifestly gauge-fixing independent quantity in Abelian theories, the infrared limit is not renormalization group invariant, and is so a useless definition even here.

The electric screening mass is correctly defined as the the position of the pole of the propagator at spacelike momentum $[2,3]$ :

$$
k^{2}+\Pi_{00}(0, \mathbf{k})=0, \quad k^{2}=-m_{s}^{2} .
$$

Here, $k=|\mathbf{k}|$. This definition is gauge fixing independent order by order in perturbation theory, which can be proved on an algebraic level [4] We also note that the two definitions normally coincide at leading order in the coupling constant. The above definition can be extended to other theories, e.g. $\phi^{4}$-theory. The polarization tensor is then replaced by the self-energy function for the scalar field, and the screening mass then reflects the screening of static scalar fields in the plasma.

However, it turns out that one cannot calculate perturbatively the screening mass beyond leading order in non-Abelian gauge theories using Eq. (3) $[2,3]$. The problem is a linear mass-shell singularity. This signals the breakdown of perturbation theory, and calls for a gauge-fixing independent and nonperturbative definition of the electric screening mass [5].

In Abelian gauge theories the above definition is equivalent to defining the Debye mass as the correlation length of equal-time electric field correlation function [5]

$$
\langle\mathbf{E}(\mathbf{x}) \cdot \mathbf{E}(0)\rangle \sim e^{-m_{s} x} / x^{3}
$$

where $x=|\mathbf{x}|$. Unfortunately, the definition Eq. (41) is a poor one in non-Abelian theories, since $\mathbf{E}$ is no longer a gauge invariant quantity. The above considerations have lead Arnold and Yaffe [5] to define the electric screening in terms of Polyakov loops. We shall not pursue this any further, but stick to the definition based on the pole of the propagator.

As is now well-known, higher order perturbative calculations at finite temperature requires resummation in order to calculate consistently in powers of the coupling constant [6]. The usual connection between the number of loops in the loop expansion and the powers of coupling constant is lost in quantum field theories at high temperature for processes where every external momentum is soft [6]. Effects of leading order in $g$ arise from every order in the loop expansion, and a resummation of the usual series of loop diagrams is required. In $\phi^{4}$ theory, resummation simply amounts to replacing the bare scalar propagator, $1 / P^{2}$, by an effective one, $1 /\left(P^{2}+m_{s}^{2}\right)$, where $m_{s}$ is a thermal mass that goes like $g T$. This remark

\footnotetext{
${ }^{1}$ The pole position is also independent of field redefinitions. Since the relation between the fields in the underlying theory and the effective theory can be viewed as a field redefinition, and since the screening mass is a long-distance quantity, one can use the effective theory to compute it. See below.
} 
also applies to Abelian gauge theories, where the one-loop self-energy diagram generates an electric screening mass for the timelike component of the gauge field. In non-Abelian gauge theories the situation is somewhat more complicated in the sense that it is also necessary to use effective vertices with a non-trivial momentum dependence, instead of the bare ones.

For the calculations of static quantities, such as free energies and screening masses, these are most conveniently carried out in the imaginary formalism, without analytic continuation to real time [7]. In this case there exists a simplified resummation scheme due to Arnold and Espinosa [8]. The point is that in the Euclidean formalism, the momentum can be soft only for $n=0$. Hence, it is only for the static modes that one uses effective propagators instead of the bare ones. Calculations based on this splitting are simpler to carry out than using the full resummation program of Braaten and Pisarski, and this approach has been used to the study phase transitions in gauge theories [8], the calculation of the free energy in e.g. $\phi^{4}$-theory and QCD $[9,10]$ as well as in the computation of the electric screening mass in QED and SQED [7].

In the study of static phenomena, such as the screening of static electric fields, there exists a convenient alternative to resummed perturbation theory, which is based on dimensional reduction and effective field theories. The idea is that the imaginary time formalism of finite temperature field theory involves free propagators in the form $\left[\mathbf{p}^{\mathbf{2}}+p_{0}^{2}+m^{2}\right]^{-1}$ where $p_{0}=2 n \pi T$ for bosons and $(2 n+1) \pi T$ for fermions and a summation over $n$. Thus for distances large compared to $1 / T$, one expects that the nonstatic modes decouple, and one is left with an effective three-dimensional field theory of the zero-frequency modes of the original fields. One strategy has therefore been to integrate out the nonstatic modes in some appproxmation. Dimensional reduction was first studied in detail in the papers by Ginsparg [11] and Landsman [12], and has later been applied by a number of authors (13] and Refs. therein).

Instead of literally integrating out the heavy modes, one may use an effective field theory approach to dimensional reduction. This method has recently been developed in an important paper by Braaten and Nieto [14]. One writes down the most general three-dimensional Lagrangian consistent with the symmetries of the system. The coefficients are determined by demanding that the static correlators in the full theory are reproduced by the ones in the effective theory at distances $R \gg 1 / T$ to some desired accuracy. Braaten and Nieto applied this method to $\phi^{4}$ theory for calculating the screening mass squared to order $g^{5} \ln g$ and the free energy to order $g^{6} \ln g$. Later they performed a calculation of the free energy in non-Abelian gauge theories [15] to order $g^{5}$, confirming the calculations of Zhai and Kastening [10]. These methods have also been applied by the present author to hot QED [16]. The calculations reproduce the results obtained by resummed perturbation theory for the free energy $[10,16]$ and the screening mass squared [7], both to order $e^{5}$.

In the present work we apply the methods of Braaten and Nieto, and the plan of the 
article is as follows. In section two we discuss SQED at high temperature and the symmetries of the effective Lagrangian. In section three, the short distance coefficients in the effective theory are determined, and in section four we compute the electric screening mass squared to order $e^{4}$. Our calculations confirm the result of Kalahsnikov and Klimov [18], who were the first to compute the screening mass to order $e^{3}$, and Blaizot et al. [7], who have derived it to order $e^{4}$ by resummation methods. In section five, we draw some conclusions, and the appendices provide the reader the necessary details as well as our conventions and notation. In the Feynman graphs a wavy line denotes the photon, a solid line the complex scalar, and a dashed line the real scalar in the effective theory.

\section{Scalar Electrodynamics at High Temperature}

The Euclidean Lagrangian of SQED is

$$
\mathcal{L}_{\mathrm{SQED}}=\frac{1}{4} F_{\mu \nu} F_{\mu \nu}+\left(\mathcal{D}_{\mu} \Phi\right)^{\dagger}\left(\mathcal{D}_{\mu} \Phi\right)+\mathcal{L}_{\mathrm{gf}}+\mathcal{L}_{\mathrm{gh}}
$$

where we have ignored the scalar self-coupling, since we will focus on the electromagnetic interactions. The covariant derivative is $\mathcal{D}_{\mu}=\partial_{\mu}+i e A_{\mu}$ and the gauge fixing term is chosen to be $\mathcal{L}_{\text {gf }}=\frac{1}{2}\left(\partial_{\mu} A_{\mu}\right)^{2}$, which is the Feynman gauge. The ghost term is then $\mathcal{L}_{\text {gh }}=\left(\partial_{\mu} \bar{\eta}\right)\left(\partial_{\mu} \eta\right)$ and is thus decoupled from the rest of the Lagrangian.

Physical quantities in SQED at high temperature receive contributions from the momentum scale $T$ which is a typical momentum of a particle in the plasma and from the momentum scale $e T$, which is the scale of electric screening. In non-Abelian theories there is also a contribution from the momentum scale $g^{2} T$, which is the order of the inverse confinement radius of QCD in three dimensions.

The two scales in scalar electrodynamics at high temperature suggests that one should integrate out the scale $1 / T$ and construct an effective three-dimensional Lagrangian, which is equivalent to SQED at momentum scales up to $e T$. We call this effective field theory electrostatic scalar quantum electrodynamics (ESQED), in analogy with the the definitions introduced by Braaten and Nieto in the case of QCD [15. This effective theory consists of a real scalar field coupled to SQED in three dimensions. The fields can be identified, up to normalizations, with the $n=0$ mode of the fields in the original theory. In particular, the real scalar field is identified with the zero-frequency mode of the time-like component of the gauge field.

Modern developments in renormalization theory implies that static correlators in SQED at long distances $R \gg 1 / T$ can be reproduced by ESQED to any desired accuracy by adding sufficiently many operators to $\mathcal{L}_{\mathrm{ESQED}}$ and tuning the coefficients as functions of $e, T$ and 
the renormalization scale $\Lambda$. The dependence of $\Lambda$ in the parameters is canceled by the $\Lambda$-dependence of loop integrals in ESQED.

Now, $\mathcal{L}_{\text {ESQED }}$ must be a gauge invariant function of the spatial fields $A_{i}$, up to the usual gauge fixing terms. This symmetry follows from the corresponding symmetry in the four dimensional theory and the Ward-Takahashi identity in the high temperature limit [12]. The breakdown of Lorentz invariance at finite temperature allows the the temporal component of the gauge field to acquire a thermal mass. Moreover, there is a three dimensional rotational symmetry and a discrete symmetry $A_{0} \rightarrow-A_{0}$. The effective Lagrangian then has the general form

$$
\begin{aligned}
\mathcal{L}_{\mathrm{ESQED}}= & \frac{1}{4} F_{i j} F_{i j}+\left(\mathcal{D}_{i} \phi\right)^{\dagger}\left(\mathcal{D}_{i} \phi\right)+M^{2}(\Lambda) \phi^{\dagger} \phi+\frac{1}{2}\left(\partial_{i} A_{0}\right)\left(\partial_{i} A_{0}\right)+ \\
& \frac{1}{2} m^{2}(\Lambda) A_{0}^{2}+e_{E}^{2}(\Lambda) A_{0}^{2} \phi^{\dagger} \phi+\mathcal{L}_{\mathrm{gf}}+\mathcal{L}_{\mathrm{gh}}+\delta \mathcal{L} .
\end{aligned}
$$

Here $\delta \mathcal{L}$ represents all other terms consistent with the symmetries. Examples of such terms are $\lambda(\Lambda) A_{0}^{4}$ and $\tilde{\lambda}(\Lambda)\left(\phi^{\dagger} \phi\right)^{2}$, which are superrenormalizable and $h(\Lambda)\left(F_{i j} F_{i j}\right)^{2}$, which is nonrenormalizable.

\section{Parameters in ESQED}

In the next subsections, we shall determine the short distance coefficients $e_{E}^{2}(\Lambda), M^{2}(\Lambda)$, and $m^{2}(\Lambda)$ We shall do so by calculating various quantities in the two theories using strict perturbation theory [14 and demanding that they match. Strict perturbation theory is simply ordinary perturbation theory in powers of $e^{2}$, neglecting resummation. In the full theory we then split the Lagrangian into a free part and an interaction part accordingly:

$$
\begin{aligned}
\left(\mathcal{L}_{\mathrm{SQED}}\right)_{0} & =\frac{1}{4} F_{\mu \nu} F_{\mu \nu}+\left(\partial_{\mu} \Phi\right)^{\dagger}\left(\partial_{\mu} \Phi\right)+\mathcal{L}_{\mathrm{gf}}+\mathcal{L}_{\mathrm{gh}} \\
\left(\mathcal{L}_{\mathrm{SQED}}\right)_{\text {int }} & =e^{2} \Phi^{\dagger} \Phi A_{\mu}^{2}-i e A_{\mu}\left(\Phi^{\dagger} \partial_{\mu} \Phi-\Phi \partial_{\mu} \Phi^{\dagger}\right)
\end{aligned}
$$

In the effective theory the mass parameters as well as $e_{E}^{2}(\Lambda)$ are of order $e^{2}$, while all other parameters are of order $e^{4}$ or higher. Thus the masses as well as higher order operators are treated as perturbations. We then write $\mathcal{L}_{\text {ESQED }}=\left(\mathcal{L}_{\text {ESQED }}\right)_{0}+\left(\mathcal{L}_{\text {ESQED }}\right)_{\text {int }}$ and strict perturbation theory corresponds to the following partition of the effective Lagrangian

$$
\begin{aligned}
\left(\mathcal{L}_{\mathrm{ESQED}}\right)_{0}= & \frac{1}{4} F_{i j} F_{i j}+\left(\partial_{i} \phi\right)^{\dagger}\left(\partial_{i} \phi\right)+\frac{1}{2}\left(\partial_{i} A_{0}\right)^{2}+\mathcal{L}_{\mathrm{gf}}+\mathcal{L}_{\mathrm{gh}}, \\
\left(\mathcal{L}_{\mathrm{ESQED}}\right)_{\text {int }}= & \frac{1}{2} m^{2}(\Lambda) A_{0}^{2}+M^{2}(\Lambda) \phi^{\dagger} \phi+e_{E}^{2}(\Lambda) \phi^{\dagger} \phi\left(A_{i}^{2}+A_{0}^{2}\right) \\
& -i e_{E}(\Lambda) A_{i}\left(\phi^{\dagger} \partial_{i} \phi-\phi \partial_{i} \phi^{\dagger}\right)+\delta \mathcal{L} .
\end{aligned}
$$


The expansion in $e^{2}$ in the full four-dimensional theory as well as in the effective field theory in three dimensions becomes increasingly infrared divergent as one goes to higher orders in the loop expansion. However, if one determines the parameters in ESQED so that it is equivalent to SQED at distances $R \gg T$, then the infrared dievergences will also be the same. This implies that one can use a strict perturbation expansion in $e^{2}$ to determine the parameters of ESQED, although the infrared problems are not treated correctly [14].

\subsection{The Coupling Constant}

For the calculation of the electric screening mass squared to order $e^{4}$, we need the gauge coupling $e_{E}$ only to leading order in $e$. By using the relation between the gauge fields in the two theories

$$
A_{i}^{3}=\sqrt{T} A_{i}
$$

and comparing $\mathcal{L}_{\mathrm{ESQED}}$ with $\int_{0}^{\beta} \mathcal{L}_{\mathrm{SQED}}$, we find

$$
e_{\mathrm{E}}^{2}(\Lambda)=e^{2} T
$$

At this order there is no dependence on the renormalization scale $\Lambda$.

\subsection{The Mass Parameters}

In this subsection we calculate the parameters $M^{2}(\Lambda)$ and $m^{2}(\Lambda)$ at leading and next-toleading order in $e^{2}$, respectively. The physical interpretation of a mass parameter is that it is the contribution to the physical screening mass from momenta of order $T$. The simplest way of determining the mass parameters is to match the screening masses in SQED and in ESQED. Denoting the self-energy for the field $\Phi$ by $\Sigma\left(k_{0}, \mathbf{k}\right)$, the scalar screening mass is the solution to the equation 2

$$
k^{2}+\Sigma(0, \mathbf{k})=0, \quad k^{2}=-m_{s}^{2} .
$$

The matching requirement implies that

$$
k^{2}+M^{2}(\Lambda)+\Sigma_{E}(k, \Lambda)=0, \quad k^{2}=-m_{s}^{2},
$$

where $\Sigma_{E}(k, \Lambda)$ is the self-energy of the field $\phi$ in the effective theory. The self-consistent solution to Eq. (10) is to leading order in the coupling constant $m_{s}^{2} \approx \tilde{\Sigma}_{1}(0)$, where $\tilde{\Sigma}_{n}\left(k^{2}\right) \equiv$ $\Sigma_{n}(0, \mathbf{k})$ denotes the $n$th order contribution to the self-energy in the loop expansion and the

\footnotetext{
${ }^{2}$ We remind the reader that this screening mass has nothing to with the screening of electric fields. As noted in the introduction, this is a quantity which gives information about the screening of static scalar fields due to rearrangements in the plasma.
} 
symbol $\approx$ is a reminder that this unphysical screening mass is obtained in strict perturbation theory. The relevant diagrams are depicted in Fig. 10 and the one-loop self-energy at zero external momentum is given by

$$
\tilde{\Sigma}_{1}(0)=(d-1) e^{2} \sum_{P} \frac{1}{P^{2}}
$$

Here, $d=4-2 \epsilon$ and the sum-integral is defined in appendix A. This immediately gives

$$
\tilde{\Sigma}_{1}(0)=\frac{e^{2} T^{2}}{4}
$$

In the effective theory the diagrams which contribute to the self-energy is shown in Fig. 2. Here the blob indicates a mass insertion. The self-energy function $\Sigma_{E}(k, \Lambda)$ vanishes in strict perturbation theory, since all the propagators are massless. Hence the matching requirement gives $m_{s}^{2} \approx M^{2}(\Lambda)$ and the mass parameter is

$$
M^{2}(\Lambda)=\frac{e^{2} T^{2}}{4}
$$

At this order $M^{2}(\Lambda)$ is independent of the scale $\Lambda$.

Let us now turn to the mass parameter $m^{2}(\Lambda)$. The screening mass is again defined as the pole of the propagator at spacelike momentum

$$
k^{2}+\Pi_{00}(0, \mathbf{k})=0, \quad k^{2}=-m_{s}^{2} .
$$

The self-energy function is given by a series expansion in $e^{2}$ and can also be expanded in a Taylor series around $k^{2}=0$. The self-consistent solution to Eq. (15) at next-to-leading order in the coupling constant is then

$$
m_{s}^{2} \approx\left[\Pi_{1}(0)+\Pi_{2}(0)\right]\left[1-\Pi_{1}^{\prime}(0)\right] .
$$

Here we have defined $\Pi\left(k^{2}\right) \equiv \Pi_{00}(0, \mathbf{k})$ and $\Pi_{n}\left(k^{2}\right)$ denotes the nth order contribution to $\Pi\left(k^{2}\right)$ in the loop expansion. The one-loop self-energy is shown in Fig 3. and equals

$$
\Pi_{1}\left(k^{2}\right)=2 e^{2} \oiint_{P} \frac{1}{P^{2}}-4 e^{2} \oiint_{P} \frac{p_{0}^{2}}{P^{2}(P+K)^{2}} .
$$

Expanding in powers of the external momentum and integrating by parts in $d-1$ dimensions yields

$$
\Pi_{1}\left(k^{2}\right)=2 e^{2} \oiint_{P} \frac{1}{P^{2}}-4 e^{2} \oiint_{P} \frac{p_{0}^{2}}{P^{4}}+\frac{4}{3} e^{2} k^{2} \oiint_{P} \frac{p_{0}^{2}}{P^{6}}+O\left(k^{4} / T^{2}\right) .
$$


The last sum-integral is ultraviolet divergent and this divergence may be removed by the wave function renormalization counterterm:

$$
Z_{\mathrm{A}}=1-\frac{e^{2}}{3(4 \pi)^{2} \epsilon}
$$

One then obtains

$$
\begin{aligned}
\Pi_{1}\left(k^{2}\right) & =\frac{e^{2} T^{2}}{3}+\frac{k^{2}}{3(4 \pi)^{2}}\left(2+2 \gamma_{E}+2 \ln \frac{\Lambda}{4 \pi T}\right)+O\left(k^{4} / T^{2}\right) \\
\Pi_{1}^{\prime}(0) & =\frac{1}{3(4 \pi)^{2}}\left(2+2 \gamma_{E}+2 \ln \frac{\Lambda}{4 \pi T}\right)
\end{aligned}
$$

We also need the self-energy at zero external momentum to two loop order. The contributing diagrams are displayed in Fig. 4 . Many of the two-loop sum-integral vanish in dimensional regularization, while others factorize into products of one-loop sum-integrals. Consider e.g. the first diagram in Fig. 田:

$$
4 e^{4} \oint_{P Q} \frac{p_{0}^{2}(P+K)^{2}}{P^{6} Q^{2}(P-Q)^{2}} .
$$

We rewrite this as

$$
4 e^{4} \mathscr{F}_{P Q}\left[\frac{2 p_{0}^{2}}{P^{6}(P-Q)^{2}}+\frac{2 p_{0}^{2}}{P^{4} Q^{2}(P-Q)^{2}}-\frac{p_{0}^{2}}{P^{6} Q^{2}}\right] .
$$

Changing variables $Q \rightarrow P-Q$ in the first term, and using the results for the two-loop sum-integrals in appendix A, we find

$$
4 e^{4} \sum_{P Q} \frac{p_{0}^{2}}{P^{6} Q^{2}}
$$

The other sum-integrals are reduced similarly and after some calculations we find

$$
\Pi_{2}(0)=-2(d-1) e^{4} \oint_{P Q} \frac{1}{P^{4} Q^{2}}+8(d-1) e^{4} \oint_{P Q} \frac{p_{0}^{2}}{P^{6} Q^{2}} .
$$

The ultraviolet divergences in the above sum-integrals actually cancel, and so we are left with a finite expression for $\Pi_{2}(0)$. This cancellation also takes place in the case of QED [16]. Using the tabulated one-loop sum-integrals in appendix A, one obtains

$$
\Pi_{2}(0)=\frac{e^{4} T^{2}}{(4 \pi)^{2}}
$$

Using these results, we finally obtain $m_{s}^{2}$ to order $e^{4}$ :

$$
m_{s}^{2} \approx \frac{e^{2} T^{2}}{3}\left[1+\left(\frac{7}{3}-\frac{2}{3} \gamma_{E}-\frac{2}{3} \ln \frac{\Lambda}{4 \pi T}\right) \frac{e^{2}}{(4 \pi)^{2}}\right] .
$$


Note that one could have obtained this result without carrying out wave function renormalization in Eq. (17). Instead one uses Eq. (16) and the divergence there is canceled by the charge renormalization counterterm.

In the effective theory the contributing diagrams are displayed in Figs. $5-6$ and again the blobs denote mass insertions. Denoting the self-energy by $\Pi_{E}(k, \Lambda)$, the screening mass is given by the solution to the equation

$$
k^{2}+m^{2}(\Lambda)+\Pi_{\mathrm{E}}(k, \Lambda)=0, \quad k^{2}=-m_{s}^{2} .
$$

All loop integrals involve massless fields and these vanish in dimensional regularization. Hence $\Pi_{E}(k, \Lambda)=0$, and so the matching relation becomes $m^{2}(\Lambda) \approx m_{s}^{2}$. Thus

$$
m^{2}(\Lambda)=\frac{e^{2} T^{2}}{3}\left[1+\left(\frac{7}{3}-\frac{2}{3} \gamma_{E}-\frac{2}{3} \ln \frac{\Lambda}{4 \pi T}\right) \frac{e^{2}}{(4 \pi)^{2}}\right]
$$

Using the renormalization group equation for the electric coupling

$$
\mu \frac{d e^{2}}{d \mu}=\frac{e^{4}}{24 \pi^{2}}+O\left(e^{6}\right)
$$

one can verify that the apparent $\Lambda$-dependence of $m^{2}(\Lambda)$ is illusory. This implies that, up to correction of order $e^{6}$, one can trade $\Lambda$ for an arbitrary renormalization scale $\mu$. The reason behind this fact is that the physical screening mass does not receive logarithmic corrections in the effective theory to order $e^{4}$ (see section 4). An important question is how to choose the arbitrary scale $\Lambda$. The mass parameter has been obtained by integrating out the nonstatic modes with masses equal to or greater than $2 \pi T$, so one expects that $\Lambda=2 \pi T$ is an appropriate choice [14]. Making this choice, the coefficient in front of $e^{2} /(4 \pi)^{2}$ takes the value $2.407 . .$, which is reasonably small.

\section{The Electric Screening Mass}

Now that we have determined the short-distance coefficients we shall use the effective threedimensional field theory and calculate the electric screening mass. We shall do so using perturbation theory and in order to take the physical effect of screening into account, we must now include the mass parameters in the free part of the effective Lagrangian. This corresponds to the following partition of $\mathcal{L}_{\text {ESQED }}$ :

$$
\begin{aligned}
\left(\mathcal{L}_{\mathrm{ESQED}}\right)_{0}= & \frac{1}{4} F_{i j} F_{i j}+\left(\partial_{i} \phi^{\dagger}\right)\left(\partial_{i} \phi\right)+M^{2}(\Lambda) \phi^{\dagger} \phi+\frac{1}{2}\left(\partial_{i} A_{0}\right)^{2} \\
& +\frac{1}{2} m^{2}(\Lambda) A_{0}^{2}+\mathcal{L}_{\mathrm{gf}}+\mathcal{L}_{\text {gh }}, \\
\left(\mathcal{L}_{\mathrm{ESQED}}\right)_{\text {int }}= & e_{E}^{2}(\Lambda) \phi^{\dagger} \phi\left(A_{i}^{2}+A_{0}^{2}\right)-i e_{E}(\Lambda) A_{i}\left(\phi^{\dagger} \partial_{i} \phi-\phi \partial_{i} \phi^{\dagger}\right)+\delta \mathcal{L} .
\end{aligned}
$$


The physical screening masses are given by the self-consistent solutions to Eqs. (11) and (27). The solution to Eq. (11) to leading order in coupling is equal to the mass parameter $M^{2}(\Lambda)$. However, recently it has been realized $[7,19]$ that the this equation has no self-consistent solution beyond leading order in perturbation theory. The problem is the last diagram in Fig. 2, which has a branch point singularity at $k=i m_{s}$. The problem is the same as in QCD, namely a scalar field interacting with a massless gauge field in three dimensions. We shall not discuss this any further, but refer to [19] where a nonperturbative definition of the scalar screening mass is discussed in detail.

The one and two-loop diagrams that contribute to the electric screening mass in ESQED are displayed in Figs. 5-6, except that the diagrams with a mass insertion are absent. We then find

$$
\begin{aligned}
\Pi_{E}(k, \Lambda)= & 2 e_{E}^{2} \int_{p} \frac{1}{p^{2}+M^{2}}-2 e_{E}^{4} \int_{p q} \frac{\delta_{i i}}{q^{2}\left(p^{2}+M^{2}\right)} \\
& +2 e_{E}^{4} \int_{p q} \frac{(p+q)^{2}}{(\mathbf{p}-\mathbf{q})^{2}\left(p^{2}+M^{2}\right)^{2}\left(q^{2}+M^{2}\right)}-2 e_{E}^{4} \int_{p q} \frac{1}{\left(q^{2}+M^{2}\right)^{2}\left(p^{2}+m^{2}\right)} \\
& -4 e_{E}^{4} \int_{p q} \frac{1}{\left(p^{2}+M^{2}\right)\left((\mathbf{p}+\mathbf{q})^{2}+m^{2}\right)\left((\mathbf{p}+\mathbf{q}+\mathbf{k})^{2}+M^{2}\right)}
\end{aligned}
$$

The integrals may be reduced to known ones by algebraic manipulations, which involve change of variables, in analogy with the calculations in the previous section. The integrals needed are tabulated in appendix B. The second integral above, which corresponds to the the first graph in Fig. 6, vanishes in dimensional regularization due to the masslessness of the photon. Moreover, the last integral is dependent on the external momentum, and the self-consistent solution to Eq. (27) at next-to-leading order in $e^{2} / m$ is found by evaluating the integral at the point $k=i m_{s}$. This can be seen from expanding the integral in powers of $k^{2}$ and noting that all terms are equally important for $k \sim e T$. The calculation of this diagram is carried out in some detail in appendix B. Notice also that the logarithmic term from this integral is exactly canceled by a corresponding term in the second two-loop integral above. Hence, there is no logarithmic correction to the electric screening mass from the effective theory through order $e^{4}$. Adding the different pieces, we obtain the physical screening mass squared to order $e^{4}$, which is the main result of the present paper:

$$
m_{s}^{2}=T^{2}\left[\frac{e^{2}}{3}-\frac{e^{3}}{4 \pi}+\frac{e^{4}}{(2 \pi)^{2}}\left(-1+\frac{1}{2 \sqrt{3}}+\left(1+\frac{\sqrt{3}}{2}\right) \ln \left(1+\frac{2}{\sqrt{3}}\right)\right)-\frac{2 e^{4}}{(12 \pi)^{2}}\left(1+\gamma_{E}+\ln \frac{\mu}{4 \pi T}\right)\right] .
$$

This result is in accordance with the calculation of Blaizot et al., who used resummation methods [7]. Using the renormalization group equation for $e$, we find that physical screening mass is independent of the renormalization scale $\Lambda$ up to corrections of order $e^{5}$.

We would also like to comment upon the $e^{3}$ term in Eq. (32). It is non-analytic in $e^{2}$, and is the first contribution to the screening mass squared from momentum scales of order 
$e T$. In resummed perturbation theory it arises from a one-loop computation which involves the dressed scalar propagator. An $e^{3}$ term is not present in QED, since there is no bosonic propagator in the corresponding one-loop graph and that fermions need no resummation because the Matsubara frequencies provide the necessary infrared cut off [16].

\section{Conclusions}

To summarize, we have derived the electric screening mass squared to order $e^{4}$ in scalar electrodynamics from an effective field theory approach. This method makes it possible to unravel the contributions to physical quantities from momentum scales of order $T$ and $e T$. This greatly simplifies calculations, since there is only a single scale involved at a time.

The running of the coupling constant first enters at order $e^{4}$ for the electric screening mass squared. Thus, an $e^{4}$ calculation is needed to determine the appropriate scale $\mu$ in the $e^{2}$ correction to this quantity. As previously argued, $\mu=2 \pi T$ is a reasonable and physically motivated choice. Finally, we would like to outline the calculations necessary to obtain the electric screening mass squared to order $e^{5}$. This requires the matching of the scalar mass parameter to two loop order, and one also has to find the coefficient for the quartic self-interaction of the temporal gauge field. In the effective theory the contribution to the screening mass squared involves the computation of three-loop diagrams.

\section{Acknowledgments}

The author is grateful to F. Ravndal for beneficial discussions.

\section{A Sum-integrals in the Full Theory}

In this appendix we give the necessary details for the sum-integrals used in present work. We closely follow the conventions of Ref. [14]. Throughout the work we use the imaginary time formalism, where the four-momentum is $P=\left(p_{0}, \mathbf{p}\right)$ with $P^{2}=p_{0}^{2}+\mathbf{p}^{2}$. The Euclidean energy takes on discrete values, $p_{0}=2 n \pi T$ for bosons. Dimensional regularization is used to regularize both infrared and ultraviolet divergences by working in $d=4-2 \epsilon$ dimensions, and we apply the $\overline{\mathrm{MS}}$ renormalization scheme. We use the following shorthand notation for the 
sum-integrals that appear below:

$$
\oiint_{P} f(P) \equiv\left(\frac{e^{\gamma_{\mathrm{E}}} \mu^{2}}{4 \pi}\right)^{\epsilon} T \sum_{p_{0}=2 \pi n T} \int \frac{d^{3-2 \epsilon} k}{(2 \pi)^{3-2 \epsilon}} f(P) .
$$

Then $\mu$ coincides with the renormalization scale in the $\overline{\mathrm{MS}}$ renormalization scheme.

The specific one-loop sum-integrals needed are listed below

$$
\begin{aligned}
\mathcal{F}_{P} \frac{1}{P^{2}} & =\frac{T^{2}}{12}\left[1+\left(2 \ln \frac{\mu}{4 \pi T}+2+2 \frac{\zeta^{\prime}(-1)}{\zeta(-1)}\right) \epsilon+O\left(\epsilon^{2}\right)\right], \\
\mathscr{f}_{P} \frac{1}{\left(P^{2}\right)^{2}} & =\frac{1}{(4 \pi)^{2}}\left[\frac{1}{\epsilon}+2 \ln \frac{\mu}{4 \pi T}+2 \gamma_{E}+O(\epsilon)\right], \\
\oiint_{P} \frac{p_{0}^{2}}{\left(P^{2}\right)^{2}} & =-\frac{T^{2}}{24}\left[1+\left(2 \ln \frac{\mu}{4 \pi T}+2 \frac{\zeta^{\prime}(-1)}{\zeta(-1)}\right) \epsilon+O\left(\epsilon^{2}\right)\right], \\
\oiint_{P} \frac{p_{0}^{2}}{\left(P^{2}\right)^{3}} & =\frac{1}{4(4 \pi)^{2}}\left[\frac{1}{\epsilon}+2 \ln \frac{\mu}{4 \pi T}+2+2 \gamma_{E}+O(\epsilon)\right] .
\end{aligned}
$$

The easiest way to evaluate the bosonic sum-integrals is to perform the integration over $k$, and then do the frequency sum by employing the integral representation of the Riemann zeta function [9]. For instance:

$$
\oiint_{P} \frac{1}{\left(P^{2}\right)^{n}}=T^{d-2 n} \frac{2(-1)^{n} \pi^{\frac{d-1}{2}}}{(2 \pi)^{2 n} \Gamma(n)} \zeta(2 n+1-d) \Gamma\left(\frac{2 n+1-d}{2}\right) .
$$

We also need some two-loop sum-integrals. They can be evaluated by contour methods [1]. Arnold and Zhai [9], and Kastening and Zhai [10] have calculated and tabulated all the sum-integrals in the list below, except for the last one.

$$
\begin{aligned}
& \mathcal{f}_{P Q} \frac{1}{P^{2} Q^{2}(P+Q)^{2}}=0, \\
& \mathcal{f}_{P Q} \frac{q_{0}^{2}}{P^{4} Q^{2}(P+Q)^{2}}=\frac{T^{2}}{12(4 \pi)^{2}}+O(\epsilon), \\
& \mathcal{F}_{P Q} \frac{p_{0}^{2}}{P^{4} Q^{2}(P+Q)^{2}}=0, \\
& \mathcal{f}_{P Q} \frac{p_{0} q_{0}}{P^{2} Q^{4}(P+Q)^{2}}=0 .
\end{aligned}
$$

The last integral can be found from the others by considering

$$
\mathcal{F}_{P Q} \frac{\left(p_{0}+q_{0}\right)^{2}}{P^{4} Q^{2}(P+Q)^{2}},
$$

and then changing variables, $Q^{\prime}=P+Q$. 


\section{B Integrals in the Effective Theory}

In the effective three-dimensional theory we use dimensional regularization in $3-2 \epsilon$ dimensions to regularize infrared and ultraviolet divergences. In analogy with Appendix A, we define

$$
\int_{p} f(p) \equiv\left(\frac{e^{\gamma_{\mathrm{E}}} \mu^{2}}{4 \pi}\right)^{\epsilon} \int \frac{d^{3-2 \epsilon} p}{(2 \pi)^{3-2 \epsilon}} f(p) .
$$

Again $\mu$ coincides with the renormalization scale in the modified minimal subtraction renormalization scheme.

In the effective theory we need the following one-loop integrals

$$
\begin{aligned}
\int_{p} \frac{1}{p^{2}+m^{2}} & =-\frac{m}{4 \pi}\left[1+\left(2 \ln \frac{\mu}{2 m}+2\right) \epsilon+O\left(\epsilon^{2}\right)\right], \\
\int_{p} \frac{1}{\left(p^{2}+m^{2}\right)^{2}} & =\frac{1}{8 \pi m}\left[1+\left(2 \ln \frac{\mu}{2 m}\right) \epsilon+O\left(\epsilon^{2}\right)\right] .
\end{aligned}
$$

All integrals are straightforward to evaluate in dimensional regularization. Details may be found in Ref. 20]. The specific two-loop integrals needed are

$$
\begin{aligned}
\int_{p q} \frac{1}{\left(p^{2}+m^{2}\right)\left(q^{2}+m^{2}\right)(\mathbf{p}-\mathbf{q})^{2}} & =\frac{1}{(4 \pi)^{2} m}\left[\frac{1}{4 \epsilon}+\frac{1}{2}\right. \\
& \left.+\ln \frac{\mu}{2 m}+O(\epsilon)\right], \\
\int_{p q} \frac{1}{\left(p^{2}+m^{2}\right)^{2}\left(q^{2}+m^{2}\right)(\mathbf{p}-\mathbf{q})^{2}} & =\frac{1}{(4 \pi)^{2} m^{2}}\left[\frac{1}{4}+O(\epsilon)\right], \\
\left.\int_{p q} \frac{1}{\left(p^{2}+M^{2}\right)\left(q^{2}+M^{2}\right)\left[(\mathbf{p}+\mathbf{q}+\mathbf{k})^{2}+m^{2}\right]}\right|_{k=i m} & =\frac{1}{(8 \pi)^{2}}\left[\frac{1}{\epsilon}+6\right. \\
\left.-4 \ln \left[\frac{2(M+m)}{\mu}\right]+4 \frac{M}{m} \ln \frac{M}{M+m}+O(\epsilon)\right] . &
\end{aligned}
$$

The two first of these integrals can be found in Ref. [15]. The integral in Eq. (B.6) has previously been calculated by Braaten and Nieto for $m=M$ in Ref. [14]. Below we compute it for the more general case $m \neq M$ using their methods. The integral can best be computed by going to coordinate space. The Fourier transform of the propagator is

$$
V_{m}(R)=\int_{p} e^{i \mathbf{p} \cdot \mathbf{R}} \frac{1}{p^{2}+m^{2}}
$$

It can be expressed in terms of a modified Bessel function

$$
V_{m}(R)=\left(\frac{e^{\gamma_{E}} \mu^{2}}{4 \pi}\right)^{\epsilon} \frac{1}{(2 \pi)^{3 / 2-\epsilon}}\left(\frac{m}{R}\right)^{1 / 2-\epsilon} K_{1 / 2-\epsilon}(m R) .
$$


In three dimensions $(\epsilon=0)$ this is the Yukawa potential:

$$
\tilde{V}_{m}(R)=\frac{e^{-m R}}{4 \pi R}
$$

For small $R$ it can be written as a sum of two Laurent series in $R^{2}$. One of these is singular beginning with an $R^{-1+2 \epsilon}$ term and the other is regular which begins with an $R^{0}$ term:

$$
\begin{aligned}
V_{m}(R)= & \left(\frac{e^{\gamma_{E}} \mu^{2}}{4}\right) \frac{\epsilon\left(\frac{1}{2}-\epsilon\right)}{\Gamma\left(\frac{1}{2}\right)} \frac{1}{4 \pi} R^{-1+2 \epsilon}\left[1+\frac{m^{2} R^{2}}{2(1+2 \epsilon)}+O\left(m^{4} R^{4}\right)\right] \\
& -\left(e^{\gamma_{E}} \mu^{2}\right)^{\epsilon} \frac{\Gamma\left(-\frac{1}{2}+\epsilon\right)}{\Gamma\left(-\frac{1}{2}\right)} \frac{1}{4 \pi} m^{1-2 \epsilon}\left[1+\frac{m^{2} R^{2}}{2(3-2 \epsilon)}+O\left(m^{4} R^{4}\right)\right] .
\end{aligned}
$$

The integral can be written

$$
\int_{p q} \frac{1}{\left(p^{2}+M^{2}\right)\left(q^{2}+M^{2}\right)\left[(\mathbf{p}+\mathbf{q}+\mathbf{k})^{2}+m^{2}\right]}=\int_{R} e^{i \mathbf{k R}} V_{M}^{2}(R) V_{m}(R)
$$

The radial integration is now split into two regions, $0<R<r$ and $r<R<\infty$. The ultraviolet divergences arise from the region $R \rightarrow 0$. This implies that we can set $\epsilon=0$ in the region where $r<R<\infty$. Hence, one can write the integral as

$$
\begin{aligned}
\int e^{i \mathbf{k R}} V_{M}^{2}(R) V_{m}(R)= & \left(\frac{e^{\gamma_{E}} \mu^{2}}{2 k}\right)^{-\epsilon} \frac{(2 \pi)^{3 / 2}}{\sqrt{k}} \int_{0}^{r} d R R^{3 / 2-\epsilon} J_{1 / 2-\epsilon}(k R) V_{M}^{2}(R) V_{m}(R)( \\
& +\frac{4 \pi}{k} \int_{r}^{\infty} d R R \sin (k R) \tilde{V}_{M}^{2}(R) \tilde{V}_{m}(R)
\end{aligned}
$$

Here, $J_{\nu}(x)$ is an ordinary Bessel function. The Bessel function has the following expansion for small $R$ :

$$
J_{1 / 2-\epsilon}(k R)=\frac{1}{\Gamma\left(\frac{3}{2}-\epsilon\right)}\left(\frac{1}{2} k R\right)^{1 / 2-\epsilon}\left[1+O\left(k^{2} R^{2}\right)\right] .
$$

Using this expansion and the small $R$ expansion of the potential, the first integral is, after dropping terms that vanish in the limit $r \rightarrow 0$

$$
\begin{aligned}
\left(\frac{e^{\gamma_{E}} \mu^{2}}{2 k}\right)^{-\epsilon} \frac{(2 \pi)^{3 / 2}}{\sqrt{k}} \int_{0}^{r} d R R^{3 / 2-\epsilon} J_{1 / 2-\epsilon}(k R) V_{M}^{2}(R) V_{m}(R)= & \frac{1}{(8 \pi)^{2}}\left[\frac{1}{\epsilon}+4 \ln \mu r\right. \\
& \left.+2+4 \gamma_{E}\right]+O(\epsilon) .
\end{aligned}
$$

The second integral can be found in e.g Ref. [21] and equals

$$
\frac{i}{2 k(4 \pi)^{2}}[(2 M+m+i k) \Gamma[-1,(2 M+m+i k) r]-(2 M+m-i k) \Gamma[(2 M+m-i k) r]] .
$$


Evaluating this at $k=i m$ yields

$$
\begin{aligned}
\left.\frac{4 \pi}{k} \int_{r}^{\infty} d R R \sin (k R) \tilde{V}_{M}^{2}(R) \tilde{V}_{m}(R)\right|_{k=i m}= & \frac{1}{(4 \pi)^{2}}\left[\frac{M}{m} \ln \frac{M}{M+m}-\gamma_{E}+1\right. \\
& -\ln [2(M+m) r]]+O(\epsilon),
\end{aligned}
$$

where we have used the series expansion of the incomplete gamma function

$$
\Gamma[-1, x]=\frac{1}{x}+\gamma_{E}-1+\ln x+O\left(x^{2}\right),
$$

and dropped terms that vanish as $r \rightarrow 0$. Collecting our results we obtain Eq. (B.6). The logarithms of $r$ cancel and our result reduces to the one found in Ref. [14] in the case $m=M$, as it should.

\section{References}

[1] J. I. Kapusta: Finite Temperature Field Theory. Cambridge University Press, Cambridge 1989.

[2] A. K. Rebhan: Phys. Rev. D 48 (1993) R3967

[3] A. K. Rebhan: Nucl. Phys. B 430 (1994) 319

[4] R. Kobes, G. Kunststatter, A. Rebhan: Nucl. Phys. B 355 (1991) 1

[5] P. Arnold, L. G. Yaffe: Phys. Rev. D 52 (1995) 7208

[6] E. Braaten, R. Pisarski: Nucl. Phys. B 337 (1990) 569

[7] J.-P. Blaizot, E. Iancu, R. Parwani: Phys. Rev. D 52 (1995) 2543

[8] P. Arnold, O. Espinosa: Phys. Rev. D 47 (1993) 3546

[9] P. Arnold, C. Zhai: Phys. Rev. D 50 (1994) 7603; Phys. Rev. D 51 (1995) 1906

[10] C. Zhai, B. Kastening: Phys. Rev. D 52 (1995), 7232

[11] P. Ginsparg: Nucl. Phys B 170 (1980) 388

[12] N. P. Landsman: Nucl. Phys. B 322 (1989) 498

[13] K. Kajantie, M. Laine, K. Rummukainen, M. Shaposhnikov: Nucl. Phys. B 458 (1996) 90 
[14] E. Braaten, A. Nieto: Phys. Rev. D 51 (1995) 6990

[15] E. Braaten, A. Nieto: Phys. Rev. D 53 (1996) 3431

[16] J. O. Andersen: Oslo Preprint 1995, Oslo-TP-95, hep-ph/9509409, to appear in Phys. Rev. D

[17] R. Parwani: Phys. Lett. B 334 (1994) 420; ERRATUM-ibid. B 342 (1995) 454

[18] O. Kalashnikov, V. V. Klimov: Phys. Lett. B 95 (1980) 423

[19] J-P Blaizot, E. Iancu: Nucl. Phys. B 459 (1996) 559

[20] L. H. Ryder: Quantum Field Theory. Cambridge University Press, Cambridge 1985.

[21] I. S. Gradshteyn, I. M. Ryzhik: Table of Integrals, Series and Products. Academic Press New York 1980. 


\section{FIGURE CAPTIONS:}

Figure 1: One-loop self-energy diagrams for the scalar field in SQED.

Figure 2: One-loop self-energy corrections for $\phi$ in ESQED.

Figure 3: One-loop self energy diagrams for the timelike photon in SQED.

Figure 4: Two-loop self energy diagrams for the timelike photon in SQED.

Figure 5: One-loop self-energy corrections for $A_{0}$ in ESQED.

Figure 6: Two-loop self-energy corrections for $A_{0}$ in ESQED. 

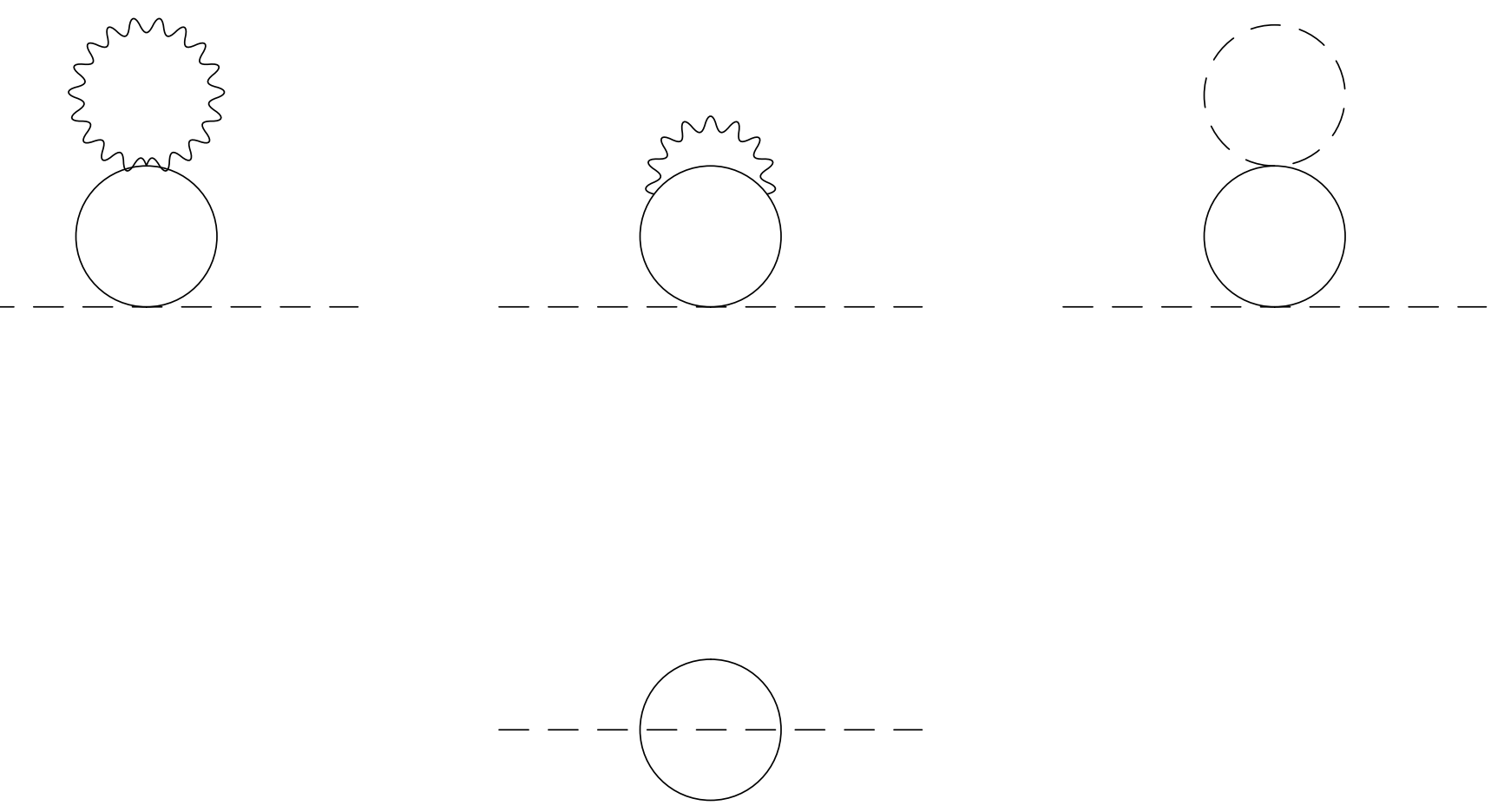
$----\bullet----$
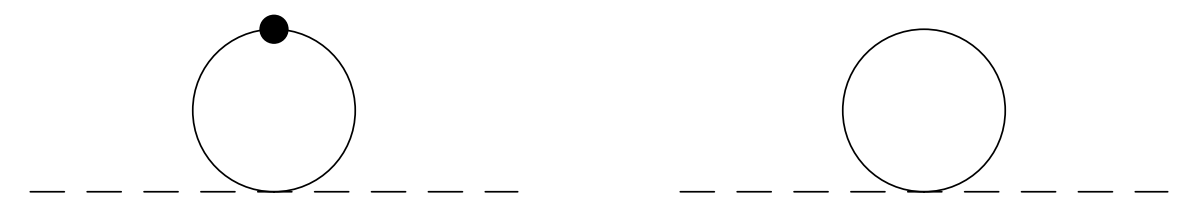

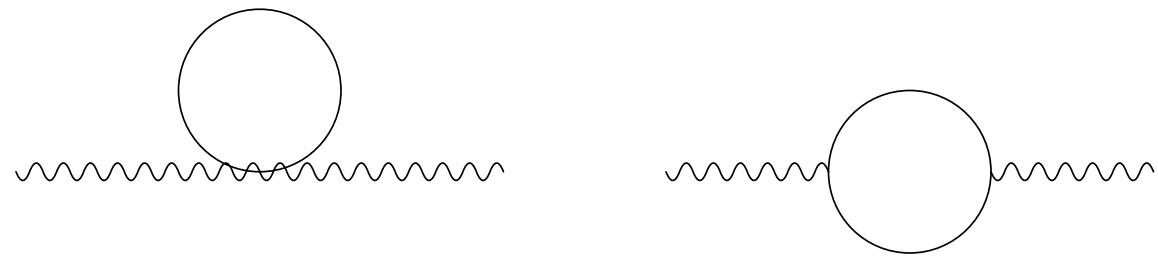

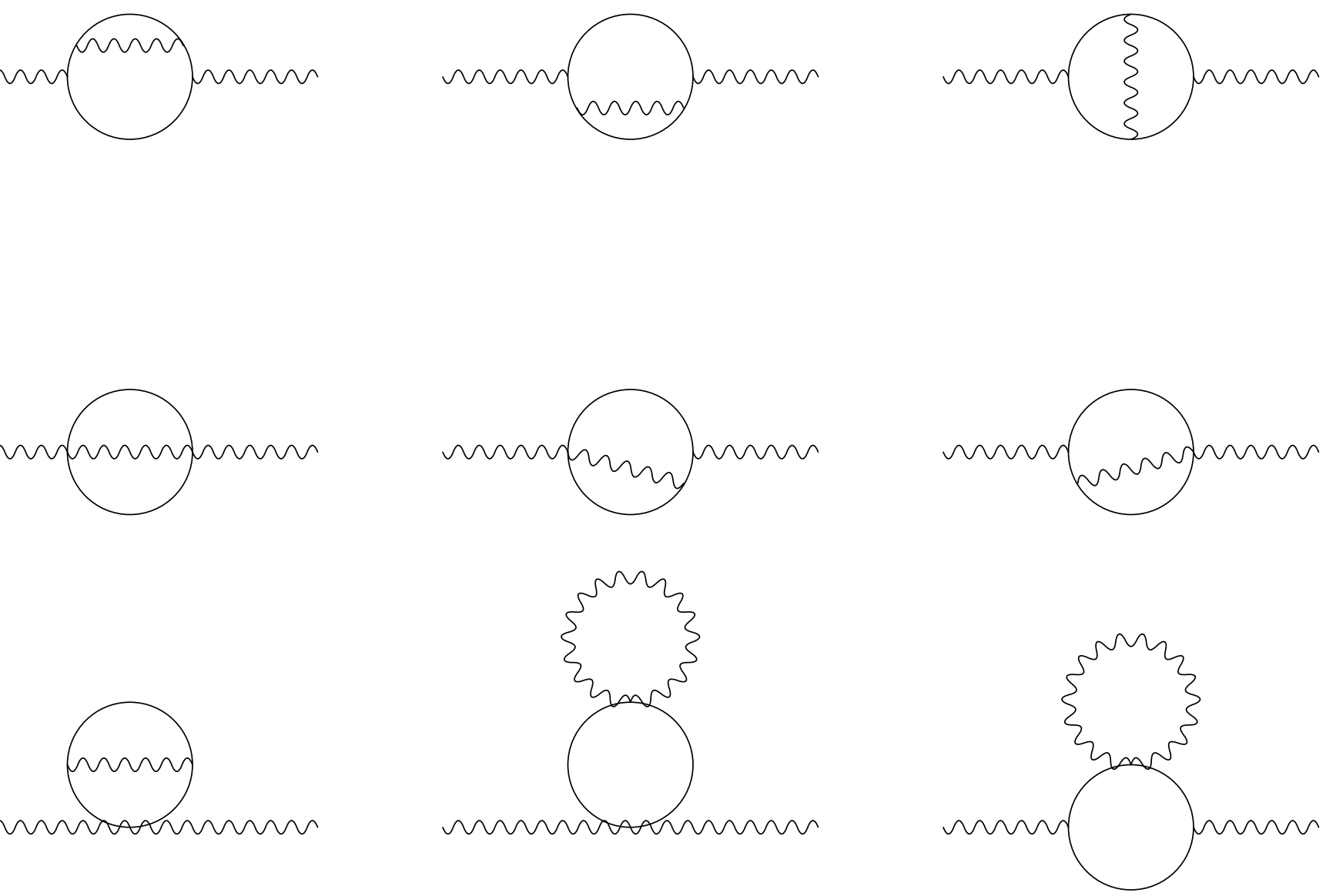

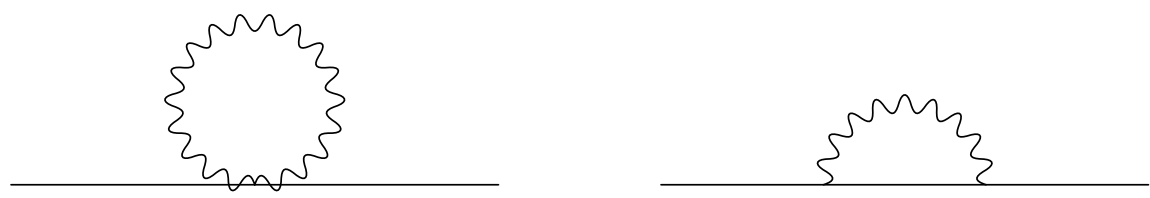

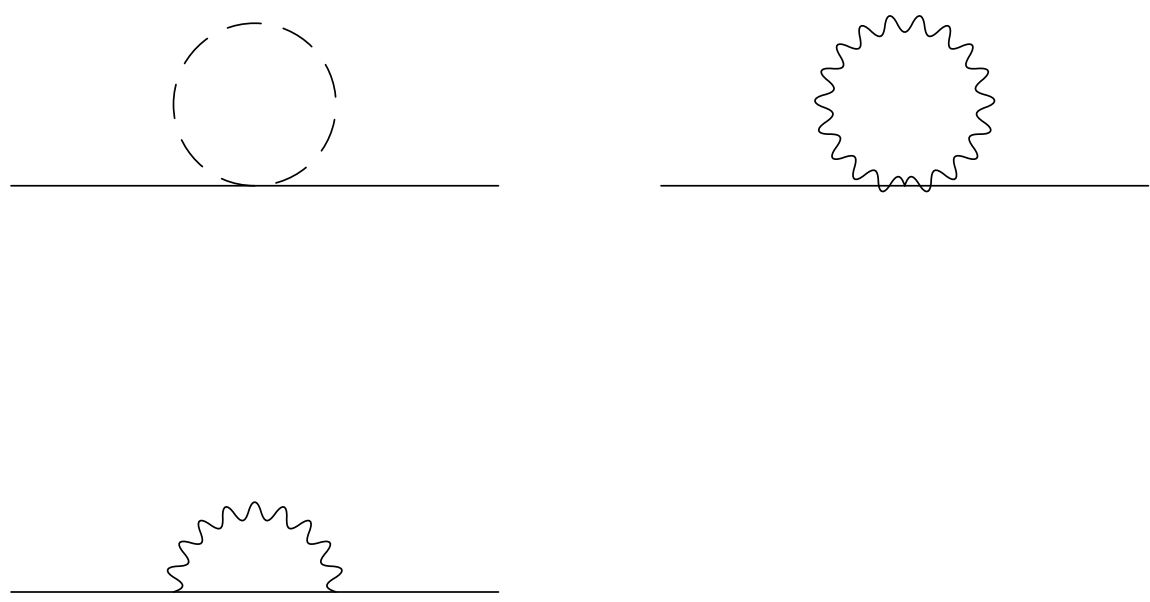